\title{
High expression level of human epidermal growth factor (hEGF) using a well-designed fusion protein-tagged construct in E. coli
}

\author{
Shams $\mathrm{D}^{1^{*}}$, Alizadeh $\mathrm{M}^{1 *}$, Azari $\mathrm{S}^{1}$, Hosseini $\mathrm{S}^{2}$, Yasami-Khiabani $\mathrm{S}^{1,3}$, Samani $\mathrm{S}^{4}$, Shokrgozar MA ${ }^{1}$
}

National Cell Bank of Iran, Pasteur Institute of Iran, Tehran, Iran. mashokrgozar@pasteur.ac.ir

\begin{abstract}
AIM: The study was aimed at design a good fusion construct that would successfully express the recombinant proteins and produce peptides in Escherichia coli. Two different constructs including human epidermal growth factor (hEGF) gene were designed to obtain an efficient expression level of hEGF. The hEGF sequence was inserted in pET32a vector containing thioredoxin (Trx) sequence and modified pET15b vector containing intein and elastin-like polypeptide (ELP).

METHODS: The vectors were transformed into $E$. coli TOP10F' for multiplication and further into $E$. coli BL21 (DE3) to express protein. The hEGF expression was induced by isopropyl $\beta$-D-1-thiogalactopyranoside (IPTG) while the expression levels were evaluated by SDS-PAGE and western blotting and compared by Image $J$ analysis, BCA and Elisa assays.

RESULTS: The expression level after 2 hours of IPTG induction was significantly higher than after other induction times. ImageJ, BCA and Elisa analyses demonstrated that the Trx presence enhanced protein expression significantly when compared to ELP-intein-based construct.

CONCLUSION: The pET32a-Trx-hEGF construct had a higher expression than pET15b-ELP-intein-hEGF.

Overall, considering Trx, the fusion protein in construct design can make it suitable to significantly express hEGF compared to ELP-intein while its combination with ELP-intein may improve the expression of the ELPintein construct (Tab. 2, Fig. 7, Ref. 34). Text in PDF www.elis.sk.

KEY WORDS: human epidermal growth factor (hEGF), thioredoxin (Trx), elastin-like polypeptide (ELP), intein, expression.
\end{abstract}

\section{Introduction}

Human epidermal growth factor is one of the most important growth factors used to induce migration, adhesion, and differentiation of various cells in tissue engineering and regenerative medicine (1). In addition, it can expedite the healing of diabetic, dermal and corneal wounds, and induce the growth of cells in different origins $(2,3)$. Consequently, an industrial production of hEGF in large-scale is necessary in support of medicine and other fields and its market demands have become enormous in recent years. In 1962, Cohen isolated EGF for the first time from mouse submaxillary gland (4) and later in 1975, he isolated the latter growth factor from human urine (5). The hEGF gene is located in chromo-

${ }^{1}$ National Cell Bank of Iran, Pasteur Institute of Iran, Tehran, Iran, ${ }^{2} \mathrm{Fac}-$ ulty of Bioscience and Biotechnology, Shahid Beheshti University, Tehran, Iran, ${ }^{3}$ Department of Biomedical Engineering, Amirkabir University of Technology, Tehran, Iran, and ${ }^{4}$ Department of Tissue Engineering and Applied Cell Sciences, School of Advanced Technologies in Medicine, Tehran University of Medical Sciences, Tehran, Iran.

"First two authors contributed equally to this article.

Address for correspondence: M.A. Shokrgozar, National Cell Bank of Iran, Pasteur Institute of Iran, Tehran, Iran.

Phone: +98.21.66492595, Fax: +98.21.66492595 some 4 and its expression leads to a single-stranded polypeptide consisting of 53 amino acids $(6.5 \mathrm{kDa})$ and three disulfide bands $(6,7)$. According to the increasing demand in the fields of medicine, nutrition industry, animal husbandry and other technologies for recombinant proteins, researchers have undergone tremendous progress to increase and optimize recombinant productions. However, the production and purification of recombinant proteins are expensive at industrial and semi-industrial levels. Therefore, it is necessary to find new ways to reduce the production costs by using recombinant DNA technology. Using simple and reliable methods to purify protein is one of the most important considerations to produce recombinant proteins (8).

E. coli as a work horse for ongoing research has some benefits such as low cost, easy technique, high growth rate and great capacity to express recombinant proteins (9). Considering these general benefits of $E$. coli and overexpression of recombinant proteins in BL21 (DE3), some studies have used it to express recombinant fusion proteins (10) and antibodies (11). Different vectors have been employed to express hEGF in E. coli $(12,13)$. Some problems related to overexpression of cloned gene products in E.coli can be overcome by inserting fusion proteins at $\mathrm{N}$ - or C-terminal of the target protein $(14,15)$. Thioredoxin (Trx) fusion protein causes oxidation-reduction of disulfide bonds in many target protein mol- 


\section{7-763}

ecules and plays an important role in the structural stability and activity of the protein $(16,17)$. Consequently, adding Trx before the target protein can enhance protein expression.

Elastin-like polypeptides (ELPs) consisting of 20-330 repeated units (Val-Pro-Gly-X-Gly, X not Pro) can undergo reversible phase transition, at the upper phase of which they become insoluble (18).The transition temperature directly depends on the type of $\mathrm{X}$ amino acids, chain length and salt concentration (19). Proteins and peptides fused to ELPs have similar temperature-responsive behavior $(18,20)$ and protein-ELP constructs allow purification of proteins from cell lysis without using chromatography columns (21). This method can be very efficient and cost-effective but ELP separation from target proteins by proteases is a major limitation. Proteases do not act specifically, and high temperature is required to activate them. This has probably a devastating effect on the stability and activity of the recombinant proteins (22). The cleavage on internal proteins (intein) and coupling two sequences of extein to each other are general processes on the cellular proteins after translation. Since this discovery, two hundred inteins with conserved sequences and lengths of 100-800 amino acids have been identified and studied (23). By using genetic engineering, different kinds of inteins have been made which can

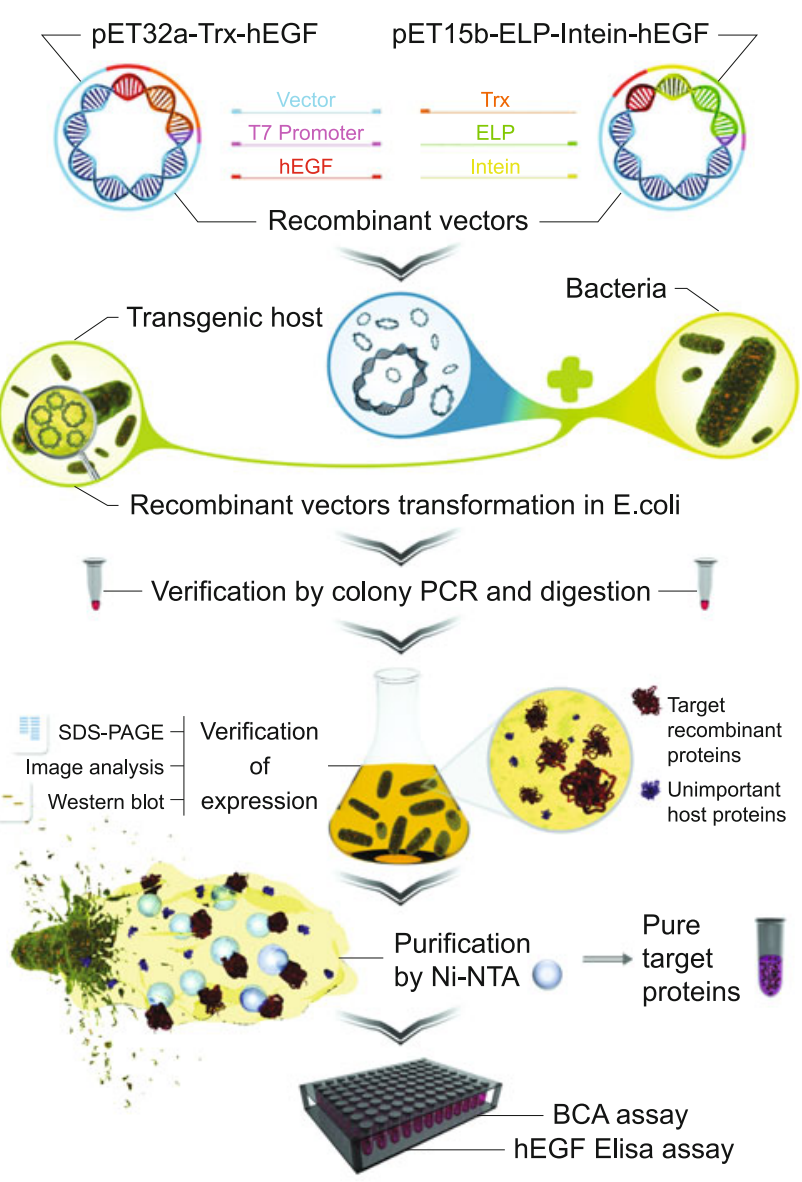

Fig. 1. Various steps of the study from design of recombinant vectors to quantitative evaluation of expressed target protein. do the cleavage from one side $(23,24)$; the cleavage between intein and the desired protein can be induced under suitable thermal and chemical ( $\mathrm{pH}$ or thiol compounds) conditions without using chromatography column and protease system (25). In this study, we designed two constructs including pET32a-Trx and modified pET15b by inserting ELP and intein to compare and introduce a suitable construct for hEGF expression in E. coli BL21. An appropriate biotechnological construct can be used for hEGF high industrial production in the future.

\section{Materials and methods}

\section{Materials}

The prokaryotic plasmid pET32a and pET15b obtained from Merck Millipore (USA). Restriction enzymes (NdeI, BamHI and XhoI), T4 DNA Ligase, DNA markers, and protein markers were purchased from TransGen Biotech (China). The colony host TOP10F', expression host BL21 (DE3), and Amicon Ultra-15 Centrifugal Filter Units were obtained from Merck Millipore (USA). Small plasmid extraction kit and Gel and PCR purification mini kit were from Yekta Tajhiz Azma (Iran). Sequencing and primer synthesis were done by Gene Fanavaran (Iran). Tris, acrylamide, bis acrylamide, ammonium persulfate, and tetramethylethylenediamine (TEMED) were purchased from Sigma-Aldrich chemical company (USA). His-tag antibody and anti-mouse HRP were from TransGen Biotech (China). BCA assay kit was purchased from Thermo-Scientific (USA) and Human EGF Standard ABTS ELISA Development Kit was obtained from PeproTech (USA).

\section{Preparation of pET15b Construct}

Figure 1 illustrates various steps of the study. ELP, intein and hEGF were cloned in pET15b according to previous study (26). Briefly, the hEGF encoding sequence was obtained from the NCBI gene bank and synthesized by Gene Fanavaran Co. The intein gene used in the $\mathrm{pET} 15 \mathrm{~b}$ construct was obtained from New England Biolabs (pTEXB1). The ELP sequence was designed to have transition temperature of about $20^{\circ} \mathrm{C}$ in the presence of 0.4 $\mathrm{M}$ sulfate amine to get precipitation. Then, the primers were designed to replicate ELP-intein-hEGF gene and get final sequence consisting of ELP (1135 bp), intein (591 bp) and hEGF (214 bp) as target protein (Fig. 2A). Finally, the ELP-intein-hEGF sequence was cloned in pUC57 vector.

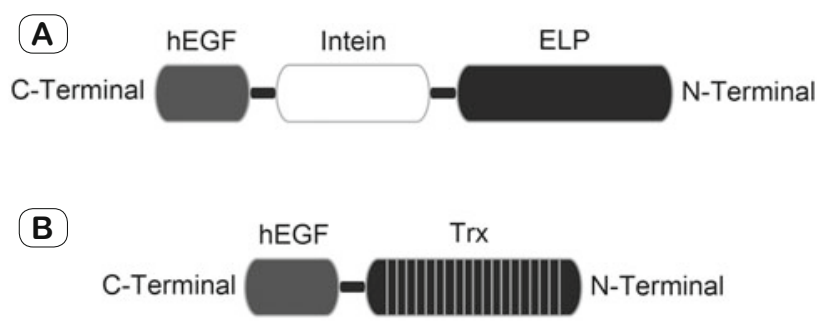

Fig. 2. Structure of (A) pET15b-ELP-intein-hEGF and (B) pET32aTrx-hEGF constructs. 
The designed ELP-intein-hEGF sequence has one restriction site for NdeI in the beginning of ELP sequence and other for BamHI at the end of the hEGF gene. After digesting the construct from pUC57 by BamHI and NdeI enzymes, the required mass of ELP-intein-hEGF was calculated by Eq.1 (27) and the inserted gene was ligated in pET15b using T4 ligase.

$$
\mathrm{V}_{\mathrm{v}}=\frac{\mathrm{T}}{\left(\frac{\mathrm{V}_{\mathrm{c}} \mathrm{I}_{1} \mathrm{I}_{\mathrm{r}}}{\mathrm{I}_{\mathrm{c}} \mathrm{V}_{1}}\right)+1} \rightarrow \mathrm{I}_{\mathrm{v}}=\mathrm{T}-\mathrm{V}_{\mathrm{v}}
$$

in which $V_{c}$ and $I_{c}$ are vector and insert concentration, $V_{1}$ and $I_{1}$ are vector and insert length, $I_{r}$ is insert-to-vector ratio. Also, $T, V_{v}$ and $I_{v}$ are volume of vector, insert and total DNA solution. Insert and vector similar parameters have same units (e.g. kilobases for length).

To clone the recombinant $\mathrm{pET} 15 \mathrm{~b}, \mathrm{TOP} 10 \mathrm{~F}^{\prime}$ became a competent cell using calcium chloride on ice while recombinant vector transformed in it by thermal shock. Then, the transgenic strain was cultured overnight on ampicillin-containing agar plate at 37 ${ }^{\circ} \mathrm{C}$. After clone selection and plasmid extraction, the presence of ELP-intein-hEGF sequence in pET15b was confirmed by digestion using NdeI and BamHI enzymes. Moreover, PCR method was used to prove the existence of intein, hEGF and intein-hEGF sequences in pET15b by employing designed primers (Tab. 1). Finally, the products of digestion and PCR were electrophoresed on $1 \%$ agarose gel.

\section{Preparation of pET32a construct}

To make the $\mathrm{pET} 32 \mathrm{a}$ construct, hEGF gene was amplified from the ELP-intein-hEGF construct by PCR technique and its primers were designed in such a way that the final construct consisted of Trx in N-terminal and hEGF in C-terminal (Fig. 2B). NEB cutter v2 online software was used to find the selective restriction enzyme sites in the desired gene and good primer design. Digested pET32a and PCR product were electrophoresed on $1 \%$ agarose gel and the vector and hEGF were extracted from agarose gel by Gel and PCR purification mini kit. The DNA ligase enzyme was used to ligate hEGF fragment and pET32a at $4{ }^{\circ} \mathrm{C}$ for 16 hours. After clone selection and plasmid extraction, same as pET15b, the presence of hEGF was confirmed in pET32a by PCR.

\section{Analyzing molecular weight of proteins}

Protein weight was analyzed by CLC main workbench v5.5 (CLC bio) to translate nucleotide sequences to amino acids and Protein Molecular Weight online software (28). As a result, the molecular weights of expressed recombinant protein in pET32a and $\mathrm{pET} 15 \mathrm{~b}$ were $21 \mathrm{kDa}$ and $61 \mathrm{kDa}$, respectively.

\section{Expression of the proteins and purification by Ni-NTA}

The constructs were extracted by plasmid extraction kit, transformed into expression host BL21 (DE3), and cultured on a solid medium containing ampicillin antibiotic. The intended clone was selected by PCR and then cultivated in $5 \mathrm{ml}$ of LB medium for 16 hours at $37^{\circ} \mathrm{C}$ while being shaken at $200 \mathrm{rpm}$. Then, $50 \mu \mathrm{l}$ of cultured clone was transferred to $100 \mathrm{ml}$ of the traffic culture (rich media). After reaching OD $=0.6$ at $600 \mathrm{~nm}$, IPTG was added at $0.5 \mathrm{mM}$ concentration, and the culture was incubated for 16 hours at $37{ }^{\circ} \mathrm{C}$ while being shaken at $200 \mathrm{rpm}$. Samples were taken after 2, 4, 6 and 16 hours of induction to determine the maximum expression of each construct. The samples before and after induction were studied by SDS-PAGE and SDS-PAGE images of two different constructs were imported to Image J software to measure the expression qualitatively (29). Also, protein expression was verified by western blot technique using mouse Anti-His antibody (1:3000 dilution in PBS containing $0.05 \%$ Tween20) and anti IgG-conjugated HRP secondary antibody (1:2000 dilution in PBS)) for one hour at $25^{\circ} \mathrm{C}$. Finally, the target band was detected by $3,3^{\prime}$-diaminobenzidine (DAB).

\section{Quantitative measurements by BCA and Elisa assay}

To measure the protein amount quantitatively, unfavorable proteins had to be removed from expressed recombinant proteins as much as possible. The bacterial culture was centrifuged, the cell pellet was lysed by sonication on ice and centrifuged for 20 min at $15000 \times \mathrm{g}(30)$. After transferring supernatant including soluble proteins (Sup1) to other tube, the pellet was resuspended in $5 \mathrm{M}$ Urea-(50 mM NaCl-200 mM Tris (NT solution)) and centrifuged to obtain inclusion proteins. Supernatant was filtered using Amicon filter by centrifuging and washed by NT solution to remove urea completely. Remaining proteins on the surface of Amicon filter were resuspended in NT solution, mixed with Sup1 and loaded on nickel resin for purification. Both constructs had His-tag at N-terminal to increase affinity with nickel resin. After incubation for $2 \mathrm{~h}$ at $4{ }^{\circ} \mathrm{C}$, the Ni-NTA column was washed twice by NT solution to remove unbound proteins. Afterwards, elute buffer (200 mM imidazole in NT solution) was added to resin, the column was centrifuged and the flow-through mixed with NT solution was filtered using Amicon filter.

BCA assay was used to determine the recombinant protein concentration because of its high accuracy and easy execution. To specify the concentration of ELP-intein-hEGF and Trx-hEGF proteins, a protein standard curve in the range of $25-2000 \mu \mathrm{g} / \mathrm{ml}$

Tab. 1. Intein and hEGF primers for PCR reaction.

\begin{tabular}{ll}
\hline Name of Primer & Primers $\left(5^{\prime}\right.$ '-----3') \\
\hline F-intein & ATGCCACGCAGGCAGGCATAGCAACAATAACAATAGCTGCATTACCGGTGATG \\
R-intein & GTTCTCGAGTTGTGAGAAACAAAACCGT \\
F-hEGF & AAGGATCCAACCAAGAAAACCCTGCGT \\
R-hEGF & ATCTCGAGTTATCATCTCAGTTCCCACCAC \\
\hline
\end{tabular}




\section{$757-763$}

was prepared. Each sample was analyzed by BCA assay kit according to manufacturer's instruction and the absorbance was measured at $562 \mathrm{~nm}$ by spectrophotometer (PowerWave XS, BioTek). Also, the amount of hEGF in the samples containing ELPintein-hEGF and Trx-hEGF proteins was quantified using hEGF ELISA assay kit. The preparation of the 96-well plate and samples as well as examination were done according to the instruction of kit's manufacturer.

\section{Statistical analysis}

A one-way ANOVA on Ranks Tukey's test post hoc $(\alpha=0.05)$ (GraphPad Prism v7.0) was carried out to assess the significant effect of IPTG induction time on protein expression in each designed construct. Also, to compare effect of construct design on protein expression after 2 hours of IPTG induction, Student's t-test ( $\alpha=$ $0.05)$ was done on Image J, BCA and Elisa data.

\section{Results}

Confirmation of ELP-intein-hEGF (pUC57 and pET15b) and hEGF (pET32a) fragments

By employing suitable primers for hEGF (F- and R-hEGF), intein (F- and R-Intein), and hEGF-intein (F-hEGF and R-iIntein),
PCR products with size of 214,591 and 805 bp were obtained, respectively. Figure $3 \mathrm{~A}$ shows related bands of hEGF, intein and intein-hEGF obtained by PCR. Also, Figure 3B depicts ELP-intein$\mathrm{hEGF}$ fragment produced by digestion of $\mathrm{pUC} 57$ and $\mathrm{pET} 15 \mathrm{~b}$. The presence of hEGF fragment in pET32a was confirmed by colony PCR method (Fig. 3C).

\section{Expression of pET32a-Trx-hEGF and pET15b-ELP-Intein-hEGF in $B L 21$}

After transforming the recombinant vectors (pET32a-TrxhEGF and pET15b-ELP-Intein-hEGF) in BL21, expression was done. According to electrophoresed gels, no expression bands were visible in pre-induction samples in the $21 \mathrm{kDa}$ and $61 \mathrm{kDa}$ regions for pET32a-Trx-hEGF and pET15b-ELP-intein-hEGF, respectively. After IPTG induction, a thick and clear band in the mentioned regions appeared according to the protein marker size (Fig. 4). Comparing the expression of the pET32a construct using Image J software showed that the expression level after 2 hours of induction by IPTG was higher than that after 4 hours of induction by $14.3 \%$ but it was not significant ( $p>0.05)$. Also, the expression after 2 hours of induction was higher than those after 6 $(\mathrm{p}<0.05)$ and 16 hours of induction $(\mathrm{p}<0.0001)$ by $15.6 \%$ and $167.5 \%$ respectively (Fig. 5A). Extra 2 hours of IPTG induction

A

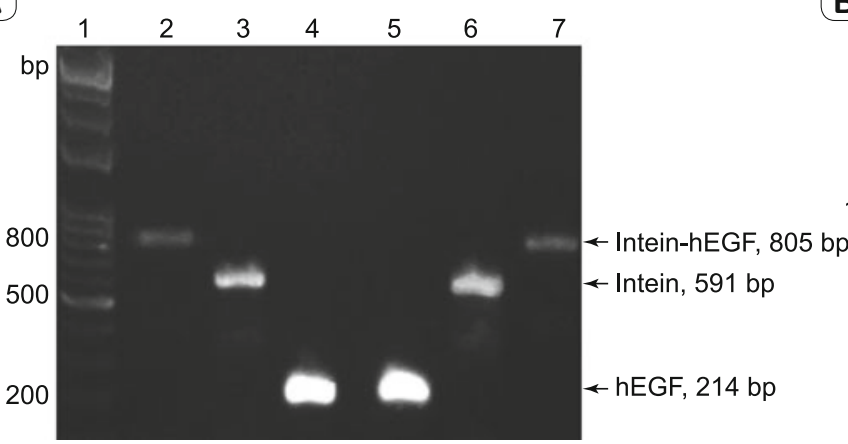

B

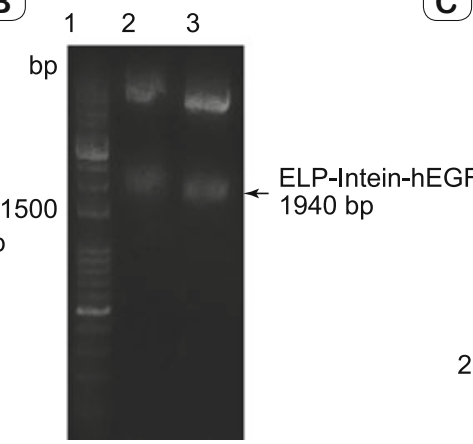

C

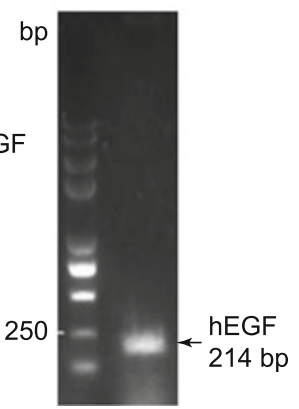

Fig. 3. Gel electrophoresis of (A) hEGF, intein and intein-hEGF obtained by PCR in pUC57 (well 2-4) and pET15b (well 5-7) (B) ELP-inteinhEGF fragment produced by digestion of pUC57 (well 2) and pET15b (well 3) and (C) hEGF fragment in pET32a.
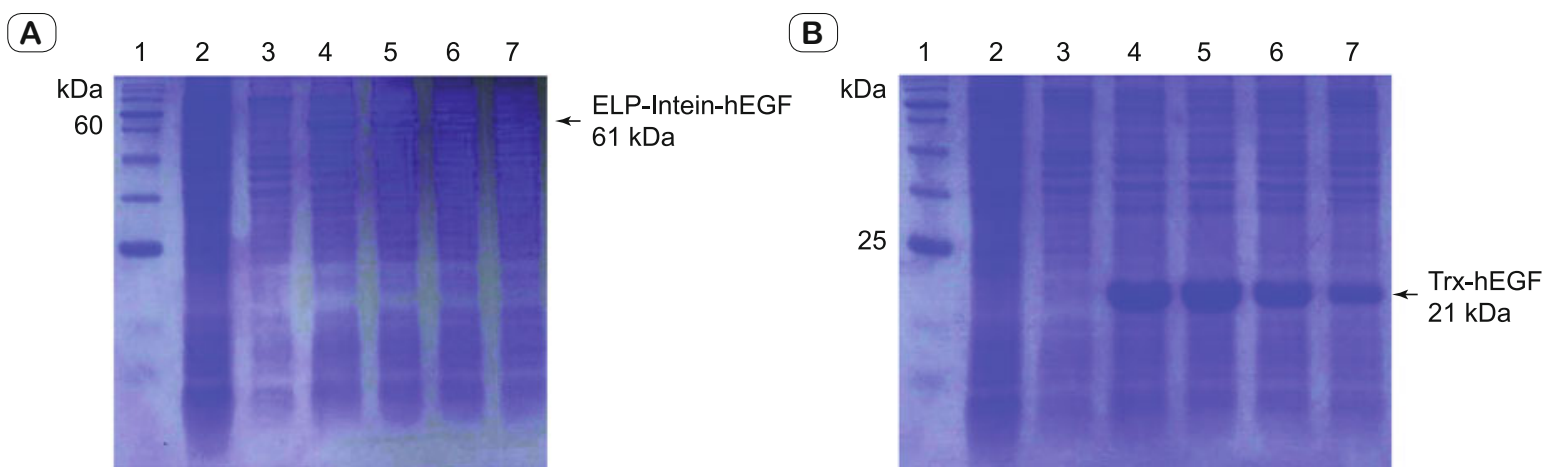

Fig. 4. The electrophoresed samples from the expression reaction in (A) pET15b and (B) pET32a constructs. Well 2: non-transgenic BL21 strain of recombinant after 4 hours and treated with IPTG; Well 3: BL21 transplanted by recombinant constructs after 4 hours and without treatment with IPTG; Wells 4-7: BL21 transplanted by recombinant pET15b and pET32a treated with IPTG for 2, 4, 6, and 16 hours indicating a protein expression of $61 \mathrm{kDa}$ and $21 \mathrm{kDa}$. 

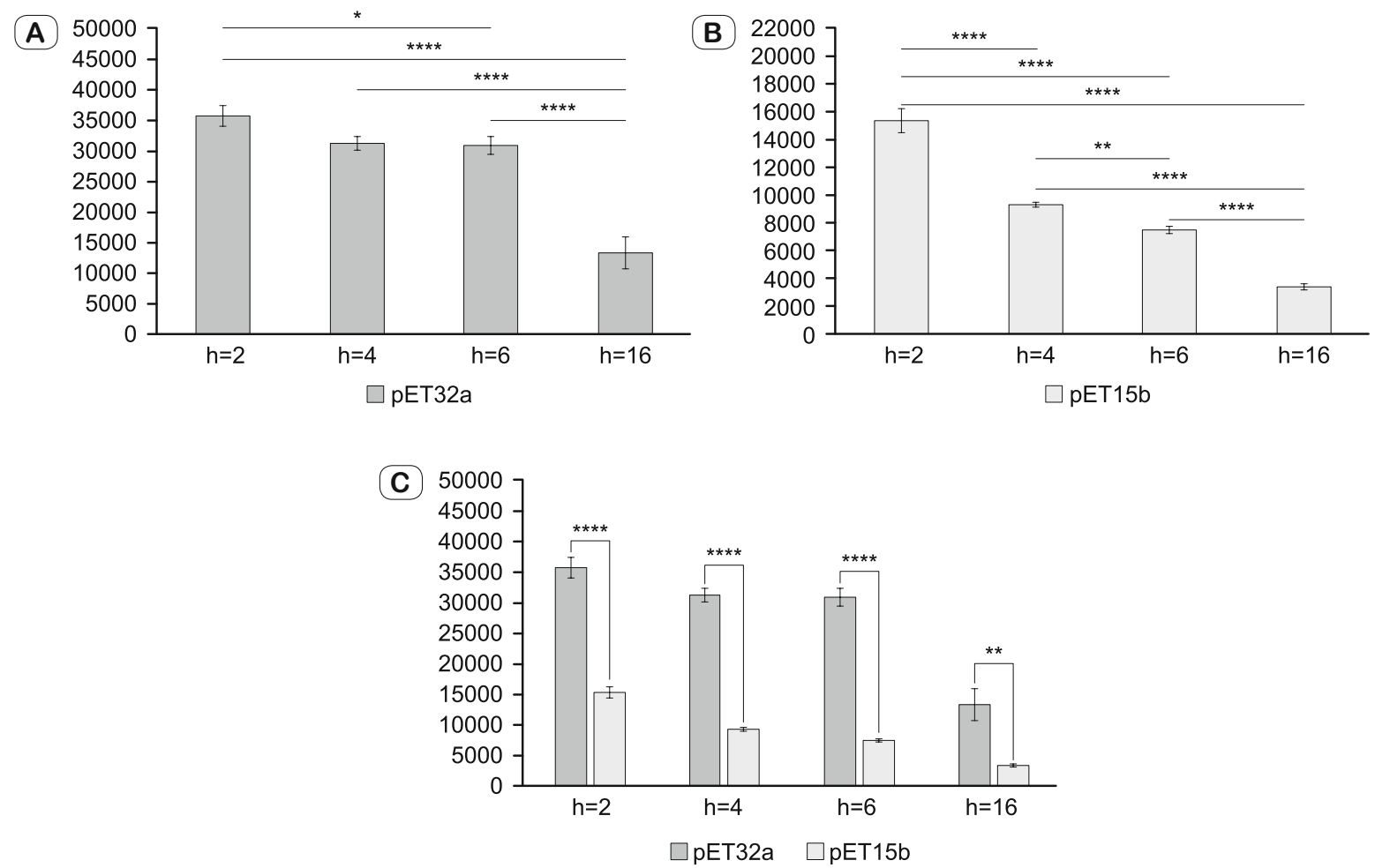

Fig. 5. Comparing the effect of various IPTG induction time on expression level by ImageJ: (A) pET32a-Trx-hEGF construct, (B) pET15bELP-Intein-hEGF and (C) between two constructs after 2 hours of induction $\left({ }^{*} \mathbf{p}<0.01, * * * * \mathbf{p}<0.0001\right)$.

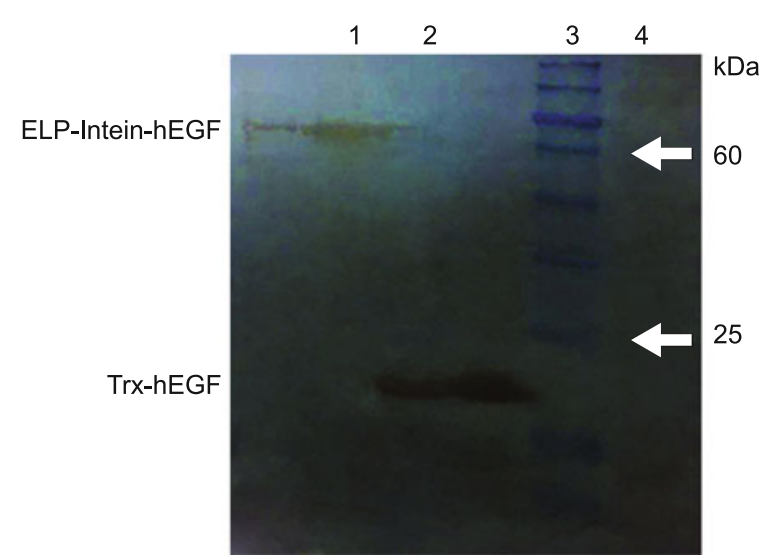

Fig. 6. Expression of target proteins in pET15b (well 1, $61 \mathrm{kDa}$ ) and pET32a (well 2, $21 \mathrm{kDa}$ ) structures by the Western blot technique. Wells 3 and 4 are related to protein marker and negative control.

(6 hours) had no significant effect on expression in comparison with the induction time of 4 hours. Fig. 5B shows that the expression of another construct (pET15b-ELP-Intein-hEGF) after 2 hours of induction was higher than those after 4,6 , and 16 hours of induction $(\mathrm{p}<0.0001)$ by $65.7 \%, 105.1 \%$, and $352.7 \%$, respectively. It must be considered that although the expression level in pET15b after 2 hours of IPTG induction was significantly better than other various induction times when compared to pET32a, the overall expression in $\mathrm{pET} 32 \mathrm{a}$ was significantly higher than that in

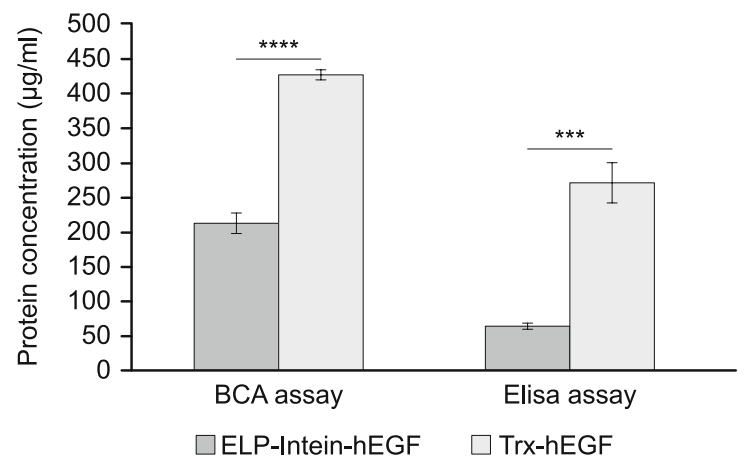

Fig. 7. Concentration of expressed protein by designed constructs measured by BCA and Elisa assay $\left({ }^{* * *} \mathrm{p}<0.001, * * * * \mathrm{p}<0.0001\right)$.

pET15b (Fig. 5C). The Table 2 includes the calculated expression difference (in percent) between different IPTG induction times in two constructs. According to ImageJ analysis, the best IPTG induction time for both constructs was 2 hours and caused a 132.3 $\%$ higher expression in pET32a-Trx-hEGF in comparison with pET15b-ELP-Intein-hEGF.

\section{Western blot assays}

Western blot analysis confirmed the expression of His-Trx$\mathrm{hEGF}$ and His-ELP-intein-hEGF proteins in BL21 using anti-his monoclonal antibody which showed reactivity of target protein (Fig. 6). 
757-763

Tab. 2. Difference in percent expression between different IPTG induction times.

\begin{tabular}{lccccc}
\hline $\mathrm{pET} 32 \mathrm{a}$ & $\mathrm{h}=16$ & $\mathrm{~h}=6$ & $\mathrm{~h}=4$ & $\mathrm{~h}=2$ & $\mathrm{pET} 15 \mathrm{~b}$ \\
\hline $\mathrm{h}=2$ & 167.5 & 15.6 & 14.3 & & $\mathrm{~h}=2$ \\
$\mathrm{~h}=4$ & 134.1 & 1.2 & & 65.7 & $\mathrm{~h}=4$ \\
$\mathrm{~h}=6$ & 131.5 & & 23.8 & 105.1 & $\mathrm{~h}=6$ \\
$\mathrm{~h}=16$ & & 120.7 & 173.2 & 352.7 & $\mathrm{~h}=16$ \\
\hline
\end{tabular}

Quantitative measurement of recombinant protein concentration

The result by BCA assay revealed that the concentration of recombinant expressed by pET32a-Trx-hEGF after 2 hours of IPTG induction was significantly higher than that expressed by pET15bELP-intein hEGF ( $<$ 0.0001) (Fig. 7) and Trx-hEGF expression was $100 \%$ higher than that of ELP-intein-hEGF.

Also, Elisa assay proved that Trx fusion to hEGF caused an increase in protein expression for pET32a-Trx-hEGF construct by $318.1 \%$ which was significantly higher than that of pET15bELP-intein-hEGF ( $\mathrm{p}<0.001)$ (Fig. 7).

\section{Discussion}

The study was aimed at comparing the expression of hEGF by two different constructs. After successful expression by using a suitable bacterial host, each recombinant protein needs to be purified at rational cost level using appropriate purification method. In traditional methods, polypeptidases are used to obtain the target recombinant protein. However, they have a non-specific cleavage function as well as low efficiency, and need high temperature to cleave the target recombinant protein (31). HPLC and Ni-NTA or other resins are different methods for purification, but on a larger scale, they have high cost in this step of production due to high column cost for HPLC and high material expenses for resins (8).

The design of the construct is the most important stage of protein production. It can influence the successful expression in host, suitable purification, and production of active native recombinant proteins. All of mentioned qualitative and quantitative improvements can be obtained by inserting tag and protein fusions (32). To decrease the financial expenses at purification step, the ELP tag can be inserted at the N-terminal of construct, which can help to separate the construct from other host proteins without using HPLC column and resins. The ELP can be sedimented under suitable conditions (temperature and salt concentration) (33), which may reduce the costs. From commercial and scientific points of view as well as when considering useful properties of intein such as self-cleavage by $\mathrm{pH}$, and specific cleavage site $(23,24)$, we designed a construct composed of ELP, intein and target protein (pET15b-ELP-Intein-hEGF) to get the native protein. Furthermore, high recombinant protein expression in host is another important point in research. Low expression level in E. coli has several reasons such as poor protein stability, protein toxicity, and rare codons within the gene. To get over the low expression level of long recombinant gene in E. coli, different approaches have been chosen by researchers. Utilizing various expression hosts or vectors, using different temperature, employing different concentration of IPTG, making short or removing the sequence from $\mathrm{N}$-terminal of long recombinant protein or gene by bioinformatics and using fusion proteins are main categories of such proposed techniques. With the exception of fusion proteins, other approaches may reduce or remove recombinant protein activity $(14,15)$. Considering oxidation-reduction function of Trx and its effect on improvement of hEGF expression in E. coli, the presence of Trx in pET32a makes it suitable to increase the expression of recombinant protein (13). Hence, another construct consisting of Trx fusion protein and hEGF (pET32a-Trx-hEGF) was designed.

The decrease in expression level of recombinant protein in both constructs after two hours of IPTG induction (Fig. 5 and Tab. 2) can be discussed from the molecular point of view. Similarly to other cells, the proliferation and growth of E. coli BL21 takes place via four stages, namely lag phase, logarithmic phase, stationary phase and death phase (34). The amounts of enzymes involved in biosynthesizing amino acids, most proteins of phospho-transferase system, some proteins involved in transportation of amino acids and peptides, and enzymes degrading less favorable substrates increase during the stationary phase in rich media (9). Because each recombinant protein is a non-specific molecule as well as a source of amino acids, it is probable that it is degraded by specific enzymes in E. coli BL21 cytoplasm, while resulting in reduction of target protein after two hours of IPTG induction.

The qualitative and quantitative analysis by ImageJ software, BCA and Elisa assays demonstrated truly that the expression level of recombinant protein increased (by $132.3 \%$ for ImageJ, $100 \%$ for BCA and $318.1 \%$ for Elisa) using pET32a-Trx-hEGF construct compared to pET15b-ELP-intein-hEGF construct in BL21 after two hours of IPTG induction. Although pET32a provides suitable conditions for high expression, it needs Ni-NTA resin and peptidases for protein purification. Thus, it is not applicable in terms of cost and pET15b-ELP-intein-hEGF fragment is cheaper and has a simple method of hEGF purification. Overall, considering the fact that the expression of this construct is lower than that of pET32aTrx-hEGF as well as on account of the effect of Trx on hEGF expression, $p E T 32 a-T r x$ in combination with ELP-intein (pET32aTrx-ELP-intein-hEGF construct) would have low cost and a simple purification route suitable for producing hEGF at a large scale.

\section{Conclusion}

This study was focused on the expression of hEGF by two constructs with different modes of design logic to introduce a suitable inexpensive expression and purification system. One construct (pET15b-ELP-Intein-hEGF) was designed considering ELP-intein sufficiency for easy and cheap purification forgoing its lower expression level. In the other construct (pET32a-Trx-hEGF), Trx fusion protein was used to enhance the expression level. Various analyses demonstrated that Trx-containing construct had a significantly higher expression level than the other. Although further studies are required to investigate the Trx effect on expression improvement of ELP-intein-hEGF in BL21, a combined construct consisting of Trx, ELP, and intein may be suggested to produce hEGF for industrial and research purposes. 


\section{References}

1. Borena BM, Martens A, Broeckx SY et al. Regenerative skin wound healing in mammals: state-of-the-art on growth factor and stem cell based treatments. Cell Physiol Biochem 2015; 36 (1): 1-23.

2. Leonida MD, Kumar I (Eds). Wound healing and skin regeneration. Switzeland: Springer, 2016.

3. Yumusak N, Yavuz U, Sarikaya B et al. Effects of epidermal growth factor on reduction of the formation of thrombus and vessel wall healing in an experimental rat model. Bratisl Med J 2017; 118 (12): 752-758.

4. Cohen S. Isolation of a mouse submaxillary gland protein accelerating incisor eruption and eyelid opening in the new-born animal. J Biol Chem. 1962; 237 (5): 1555-1562.

5. Cohen S, Carpenter G. Human epidermal growth factor: isolation and chemical and biological properties. P Natl Acad Sci USA. 1975; 72 (4): 1317-1321.

6. Razis AFA, Ismail EN, Hambali $\mathbf{Z}$ et al. The periplasmic expression of recombinant human epidermal growth factor (hEGF) in Escherichia coli. Asia-Pac J Mol Biol Biotechnol 2006; 14 (2): 41-45.

7. Su Z, Huang Y, Zhou Q et al. High-level expression and purification of human epidermal growth factor with SUMO fusion in Escherichia coli. Protein Peptide Lett 2006; 13 (8): 785-792.

8. Fong BA, Wu WY, Wood DW. The potential role of self-cleaving purification tags in commercial-scale processes. Trends Biotechnol 2010; 28 (5): $272-279$.

9. Li Z, Nimtz M, Rinas U. The metabolic potential of Escherichia coli BL21 in defined and rich medium. Microb Cell Fact 2014; 13 (1): 45-61.

10. Hekmat $S$, Siadat $S$, Aghasadeghi $M$ et al. From in-silico immunogenicity verification to in vitro expression of recombinant Core-NS3 fusion protein of HCV. Bratisl Med J 2017; 118 (4): 189-195.

11. Hashemi M, Amirijavid S, Entezari M et al. Generation and characterization of chicken egg yolk antibodies (IgY) against TNFR1. Bratisl Med J 2015; 116 (5): 316-320.

12. Gasparian ME, Ostapchenko VG, Schulga AA et al. Expression, purification, and characterization of human enteropeptidase catalytic subunit in Escherichia coli. Protein Expr Purif 2003; 31 (1): 133-139.

13. Soler LF, Cedano J, Querol E et al. Cloning, expression and purification of human epidermal growth factor using different expression systems. J Chromatogr B 2003; 788 (1): 113-123.

14. Lee MS, Lin IF, Lai GH et al. Statistical optimization of culture medium for the overproduction of chicken anemia virus immunogen-VP1 protein in a recombinant E. coli for vaccine application. Asia-Pac J Chem Eng 2015; 10 (1): 96-104.

15. Lee MS, Hseu YC, Lai GH et al. High yield expression in a recombinant $\mathrm{E}$. coli of a codon optimized chicken anemia virus capsid protein VP1 useful for vaccine development. Microb Cell Fact 2011; 10 (1): 56-67.

16. Aispuro-Hernandez E, Garcia-Orozco KD, Muhlia-Almazan A et al. Shrimp thioredoxin is a potent antioxidant protein. Comp Biochem Physiol C Toxicol Pharmacol 2008; 148 (1): 94-99.
17. Demain AL, Vaishnav P. Production of recombinant proteins by microbes and higher organisms. Biotechnol Adv 2009; 27 (3): 297-306.

18. Hassouneh W, Christensen T, Chilkoti A. Elastin-like polypeptides as a purification tag for recombinant proteins. Curr Protoc Protein Sci 2010; 61 (1): 6-11.

19. MacEwan SR, Chilkoti A. Elastin-like polypeptides: Biomedical applications of tunable biopolymers. Biopolymers 2010; 94 (1): 60-77.

20. Zhao B, Lindeboom T, Benner $S$ et al. Predicting the Fluid-Phase Behavior of Aqueous Solutions of ELP(VPGVG) Sequences Using SAFTVR. Langmuir 2017; 33 (42): 11733-11745.

21. Banki MR, Feng L, Wood DW. Simple bioseparations using selfcleaving elastin-like polypeptide tags. Nat Methods 2005; 2 (9): 659-661.

22. Young CL, Britton ZT, Robinson AS. Recombinant protein expression and purification: a comprehensive review of affinity tags and microbial applications. Biotechnol J 2012; 7 (5): 620-634.

23. Muralidharan V, Muir TW. Protein ligation: an enabling technology for the biophysical analysis of proteins. Nat Methods 2006; 3 (6): 429-438.

24. Frutos S, Goger M, Giovani B et al. Branched intermediate formation stimulates peptide bond cleavage in protein splicing. Nat Chem Biol 2010; 6 (7): 527-533.

25. Xu MQ, Evans TC. Recent advances in protein splicing: manipulating proteins in vitro and in vivo. Curr Opin Biotech 2005; 16 (4): 440-446.

26. Omoumi N, Shokrgozar MA, Noormohammadi Z. Design and Construction of Recombinant ELP-Intein Cassette for Use in Simple and new Purification Methods of Recombinant Proteins. Mod Med Lab J 2017; 1 (2): 84-90.

27. Cranenburgh RM. An equation for calculating the volumetric ratios required in a ligation reaction. Appl Microbiol Biot 2004; 65 (2): 200-202.

28. Protein Molecular Weight [https://www.bioinformatics.org/sms/ prot mw.html]

29. Nasiri M, Babaie J, Amiri S et al. SHuffle ${ }^{\mathrm{TM}} \mathrm{T} 7$ strain is capable of producing high amount of recombinant human fibroblast growth factor-1 (rhFGF-1) with proper physicochemical and biological properties. J Biotechnol 2017; 259: 30-38.

30. Esipov RS, Stepanenko VN, Chupova LA et al. Production of recombinant human epidermal growth factor using Ssp dnaB mini-intein system. Protein Expr Purif 2008; 61 (1): 1-6.

31. Waugh DS. An overview of enzymatic reagents for the removal of affinity tags. Protein Expr Purif 2011; 80 (2): 283-293.

32. LaVallie ER. Production of recombinant proteins in Escherichia coli. Curr Protoc Protein Sci 1995; 1 (1): 1-5.

33. Shi C, Meng Q, Wood DW. A dual ELP-tagged split intein system for non-chromatographic recombinant protein purification. Appl Microbiol Biot 2013; 97 (2): 829-835.

34. Smith A, Kaczmar A, Bamford RA et al. The culture environment influences both gene regulation and phenotypic heterogeneity in Escherichia coli. Front Microbiol 2018; 9: 1739-1751.

Received March 4, 2019. Accepted July 15, 2019. 